\title{
Blood clot stabilization on root dentin conditioned by the combination of tetracycline and EDTA
}

\author{
Gustavo Giacomelli Nascimento ${ }^{1}$, Amauri Antiquera Leite ${ }^{2}$, Elza Regina Manzolli Leite ${ }^{2}$, \\ José Eduardo Cezar Sampaio ${ }^{3}$, Fábio Renato Manzolli Leite ${ }^{1}$
}

\author{
${ }^{1}$ Universidade Federal de Pelotas - UFPel, School of Dentistry, Department of Semiology and Clinics, Pelotas, RS, Brazil \\ ${ }^{2}$ Universidade Estadual Paulista - UNESP, School of Pharmaceutical Sciences, Department of Clinical Analysis, Araraquara, SP, Brazil \\ ${ }^{3}$ Universidade Estadual Paulista - UNESP, Araraquara Dental School, Department of Diagnosis and Surgery, Araraquara, SP, Brazil
}

Received for publication: January 24, 2014 Accepted: March 25, 2014

Correspondence to: Fábio Renato Manzolli Leite Universidade Federal de Pelotas Faculdade de Odontologia Departamento de Semiologia e Clínica Rua Gonçalves Chaves, CEP 96015-560

Centro, Pelotas, RS, Brasil

Phone: +55 5332256741

E-mail: leite.fabio@odontoufpel.com.br

\begin{abstract}
Aim: To assess the combined use of tetracycline (TTC) and ethylenediaminetetraacetic acid (EDTA) on clot formation, considering that EDTA may neutralize TTC acidity. Methods: Planed human tooth roots were treated with saline solution, EDTA, TTC and their combination (EDTA followed by TTC and TTC before EDTA). Fresh human blood was applied on the conditioned surfaces to check clot adhesion and stabilization. A previously calibrated (kappa $=0.93)$ and blinded examiner scored scanning electron micrographs of the samples. Statistical analyses were performed using one-way ANOVA and Tukey's test. Results: Application of TTC before EDTA presented the best results with the highest number of cells adhered to the root surface $(p=0.046)$. Use of EDTA alone and EDTA before TTC disturbed clot stabilization when compared to control group $(p<0.01)$. Conclusions: The use of TTC before EDTA seems to be able to keep blood cells viable to establish an organized clot and could be used by clinicians together with the conventional mechanical root scaling and planing.
\end{abstract}

Keywords: ethylenediaminetetraacetic acid; periodontal regeneration; scanning electron microscopy; smear layer.

\section{Introduction}

One of the main goals of periodontal therapy is to obtain new connective tissue attachment or reattachment to roots already exposed to oral environment ${ }^{1}$. Histological and ultrastructural studies have demonstrated that dental roots exposed to the oral cavity or to periodontal pockets present reduced collagen fiber insertion ${ }^{2}$, change their mineral density and contaminate them by bacteria and their products. Scaling and root planing are the best techniques for mechanical decontamination. However, these methods per se are not able to fully eliminate the etiological contaminants, and they produce a compact smear layer that covers the instrumented surface inhibiting periodontal reattachment ${ }^{3}$. For these reasons, the use of demineralizing agents has been studied as an adjunct to periodontal therapy, since these agents present a great potential not only for removing smear layer ${ }^{4}$, but also to expose the underlying radicular collagen fibrils and to restore the biocompatibility of the roots ${ }^{5}$. These properties are necessary to increase fibrin 
network linkage to root collagen fibers, resulting in a better periodontal regenerative procedure ${ }^{4,6}$.

Some reports have demonstrated that demineralization of the root surface could not only exert neutralizing effects on endotoxins from periodontal pathogens, but also induce fibroblast proliferation, synthesis and attachment ${ }^{7}$. Additionally, studies have shown that in comparison to nonconditioned teeth, acid conditioned tooth roots presented a greater tendency to maintain fibrin clot, exposing collagen fibrils and increasing the levels of proteoglycans ${ }^{8}$.

An improvement of biological response on root surface after conditioning with demineralizing agents has been described ${ }^{9}$. In vivo human ${ }^{9}$ histological studies have shown improved biological response when decalcifying/chelating agents are used to condition the root surface. Citric acid, phosphoric acid, ethylenediaminetetraacetic acid (EDTA) and tetracycline hydrochloride (TTC) ${ }^{10}$ are considered the most used agents for this purpose. Despite the well-described uses of these agents, there is lack of standardization of the procedures employed by clinicians and researchers. Thus, the great variability of protocols has avoided reliable comparisons among them.

Moreover, there is only one systematic review about this subject, published by Mariotti in $2003^{11}$. The author concluded that use of citric acid, TTC or EDTA to modify the root surface provided no clinically significant benefit for regeneration in patients with chronic periodontitis ${ }^{11}$. Nevertheless, several factors such as lack of controls, noncalibrated examiners, masked reference standards and small sample sizes among others, reduced the quality of relevant studies, so the conclusions of Mariotti's review must be carefully considered.

Many studies showed that EDTA might have controversial results on tissue repair, since its use could disturb clot formation and TTC may kill connective tissue cells due to its acid $\mathrm{pH}$. Thus, the aim of this study was to compare the influence of root conditioning with TTC, EDTA and their combination on blood elements adsorption and adhesion to root surfaces. The main goal was to check the possible combined use of TTC with EDTA on clot stabilization, since EDTA may neutralize TTC acidity.

\section{Material and methods}

This study was approved by the Research Ethics Committee of the School of Dentistry at Araraquara, UNESP - Univ Estadual Paulista (Protocol \#10/04). All patients signed an informed consent. Freshly extracted human teeth with periodontal disease characterized by at least $6 \mathrm{~mm}$ attachment loss and bleeding on gentle probing, absence of caries or restorations below the cementoenamel junction ${ }^{4}$ were used. Teeth were individually placed in capped tubes containing saline solution.

\section{Sample preparation:}

The cervical third of roots was chosen for sample preparation. Specimens were prepared by making two parallel 0.5-mm-deep grooves on the buccal and lingual root surfaces using a high-speed cylindrical bur (KG Sorensen, Barueri, $\mathrm{SP}$, Brazil) under copious irrigation. One groove was made at the cementoenamel junction and the other approximately $3 \mathrm{~mm}$ distant from this point, in the apical direction. The same bur was used to remove the surface layer of the root between the two grooves. The area between grooves was then scaled with 50 apical-cervical strokes using a sharp \#56 Gracey curette (Hu-Friedy, Chicago, IL, USA) in order to create a smear layer. Samples were stored in containers with saline solution $(n=50)$.

Specimens were divided into five groups $(n=10): 1)$ irrigation with $10 \mathrm{~mL}$ of saline solution (control - GI); 2) fresh tetracycline hydrochloride $25 \mathrm{mg} / \mathrm{mL}$ (Valde Química, São Paulo, SP, Brazil) (TTC - GII) ${ }^{12} ; 3$ ) application of $24 \%$ EDTA PrefGel (Biora AB, Malmö, Sweden) (GIII); 4) TTC used before EDTA (GIV); 5) EDTA used before TTC (GV). Substances were applied with a soft brush for $3 \mathrm{~min}$, changing solutions every $30 \mathrm{~s}$. Immediately after, teeth were washed with $10 \mathrm{~mL}$ of sterile saline solution. Fresh human blood from a healthy male donor was placed on dentin surface and allowed for $20 \mathrm{~min}$ to form clot in a humidified chamber at $37{ }^{\circ} \mathrm{C}$ (Figure 1). Specimens were then rinsed three times for 5 min in PBS. Washes and rinses of the root specimens were performed in small Petri dishes with a gentle swirling motion using a rotating tabletop shaker at low speed.

After rinsing, the specimens were fixed in $1 \%$ formaldehyde diluted in PBS for $15 \mathrm{~min}$. After three 5-min PBS rinses, the samples were incubated for $10 \mathrm{~min}$ in $0.02 \mathrm{M}$ glycine diluted in PBS and rinsed again. Specimens were post-fixed in $2.5 \%$ glutaraldehyde in PBS for $30 \mathrm{~min}$ and rinsed again. Dehydration was performed by immersion in an increasing ethanol series: 30\%, 50\%, 75\%, 90\%, 95\% and three exchanges of $100 \%$. The specimens were dried in a carbon dioxide critical point drier (Baltec CPD 030; BalTec Union Ltd., Liechtenstein) and then dried at room temperature. Samples were mounted on aluminum stubs with colloidal graphite, sputter coated with gold palladium in a specified device (Baltec SCD 050, Bal-Tec Union Ltd.,), and stored and desiccated at room temperature for 3 days ${ }^{10}$.

\section{SEM examination:}

SEM micrographs $(500 \times$ and $1000 \times)$ from the central area of each specimen were obtained in a scanning electron microscope (JEOL JSM-T330A; Miaka, Tokyo, Japan) adjusted to $20 \mathrm{kV}$ and evaluated by a trained and calibrated (kappa $=0.93$ ) examiner blinded to the experimental groups ${ }^{10,12}$. SEM micrographs of the specimens that received blood application were evaluated by the blood elements adhesion index (BEAI $)^{10}$ shown in Figure 2:

Score 0: Absence of fibrin network and blood cells.

Score 1: Scarcely distributed fibrin network and/or blood cells.

Score 2: Moderate number of blood cells and thin fibrin network with poor interlacing.

Score 3: Dense fibrin network with rich interlacing and presence of blood cells. 


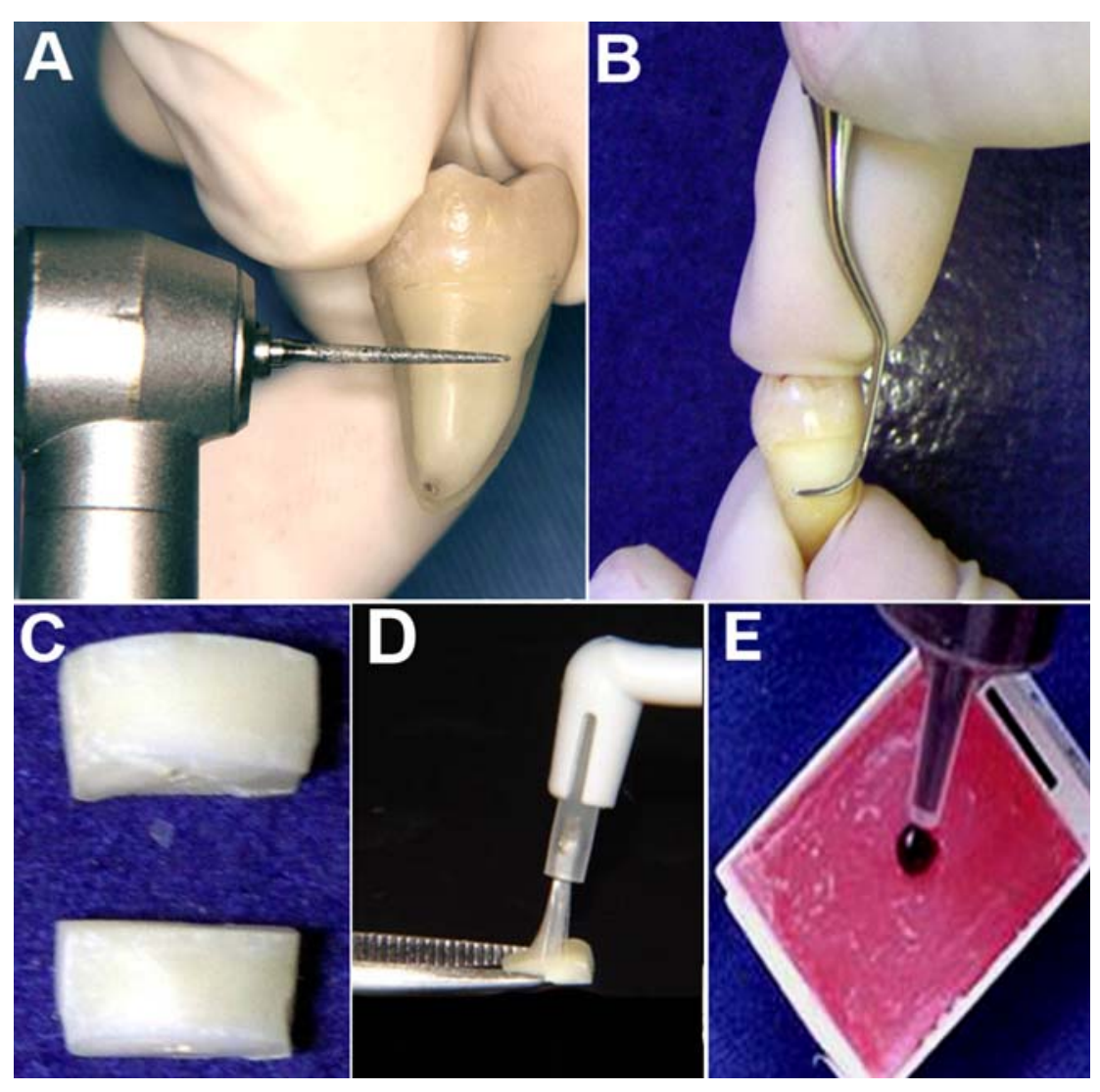

Fig. 1 - Sample preparation. A: Parallel grooves; B: Root scaling; C: Dentin samples; D: Solution application with soft brush; E: Blood drop allowed to clot.

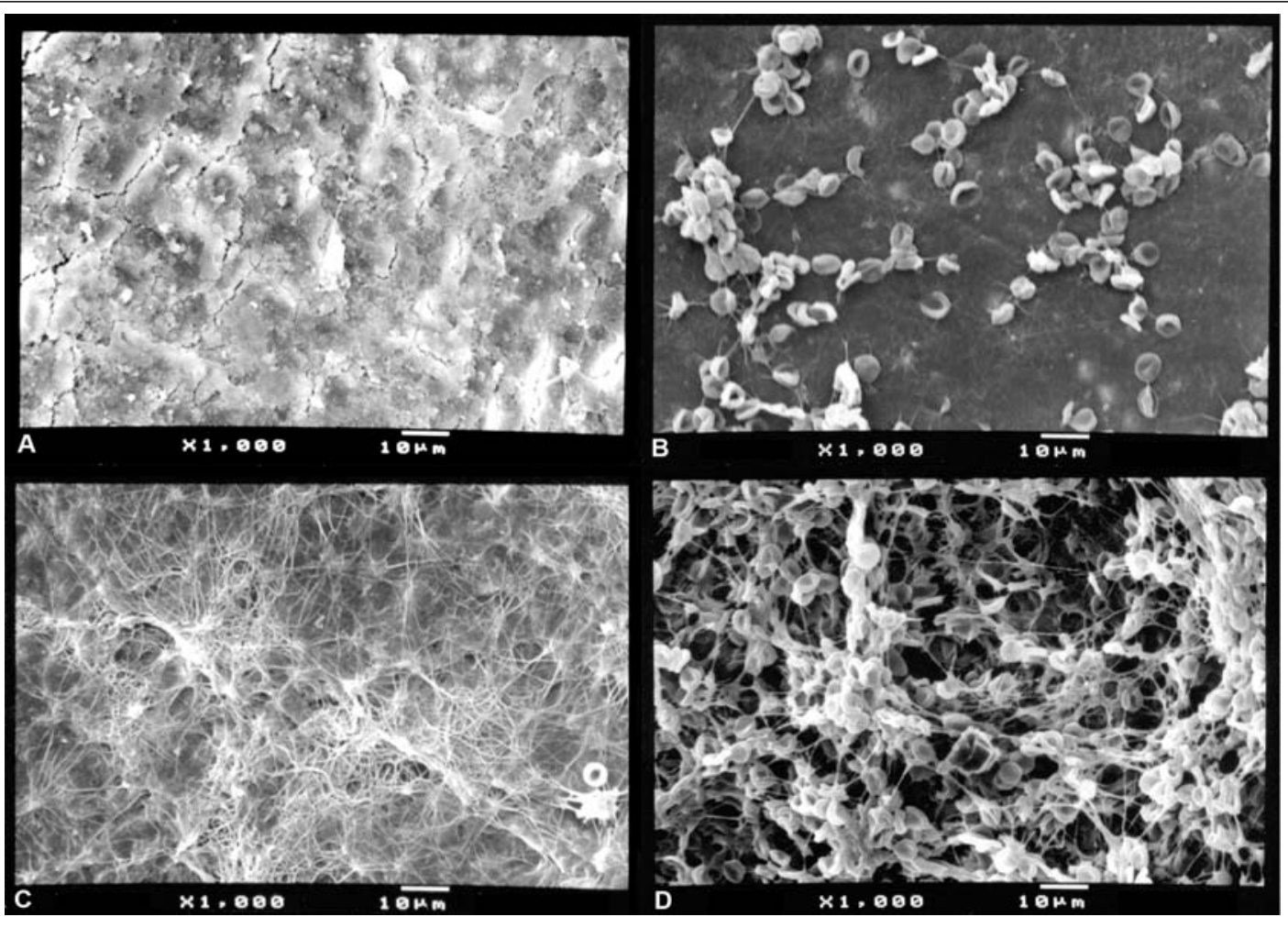

Fig. 2 - Blood Elements Adhesion Index (BEAl) score. A: Absence of fibrin network and blood cells (score 0); B: Scarcely distributed fibrin network and/or blood cells (score 1); C: Moderate number of blood cells and thin fibrin network with poor interlacing (score 2); D: Dense fibrin network with rich interlacing and presence of blood cells (score 3). 
Each SEM micrograph was evaluated three times in intervals of at least 7 days. The score attributed to each sample was the predominant score in the three evaluations.

\section{Statistical analysis}

BEAI results for each group were compared by one-way ANOVA and Tukey's test at a significance level of $p \leq 0.05$. Analyses were performed using the GraphPad Instat Software 5.05 (GraphPad Software, Inc., San Diego, CA ).

\section{Results}

GIV presented the best results among the groups, with $80 \%$ of the specimens presenting scores 2 and 3 . Sixty percent of the specimens in the control group (GI) received score 2. Frequency distribution according to groups and scores is exhibited in Table 1.

A great variation in morphological aspects of fibrin network and blood cells amount was observed. Analyzing separately the scores of each group, the samples of GIII and GV had a similar distribution of scores, showing that these treatments are able to provide homogeneous final results. In GI and GII the scores ranged from 0 to 2 .

ANOVA test rejected the hypothesis that all groups have the same performance in clot stabilization $(p<0.001)$. Variation among groups' means was significantly greater than expected by chance. EDTA presented the worst results regarding clot organization compared to all groups. The use of TTC before EDTA increased the clot stabilization potential $(p=0.046)$ and TTC before EDTA improved clot organization when compared to the use of TTC after EDTA $(p<0.05)$. EDTA alone and EDTA before TTC conditioning disturbed clot stabilization compared to control group $(p<0.01)$. Figure 3 summarizes ANOVA and Tukey test results.

\section{Discussion}

To the best of our knowledge, this is the first study to evaluate the effects of EDTA and TTC used in combination. Both agents are the most used for conditioning root surface adjunct to conventional periodontal treatment. As previously described, scaling and root planing are unable to decontaminate completely the root surface and produce a smear layer ${ }^{13}$ formed by remnants of calculus, plaque and contaminated dental hard tissues (cement and dentin). Smear

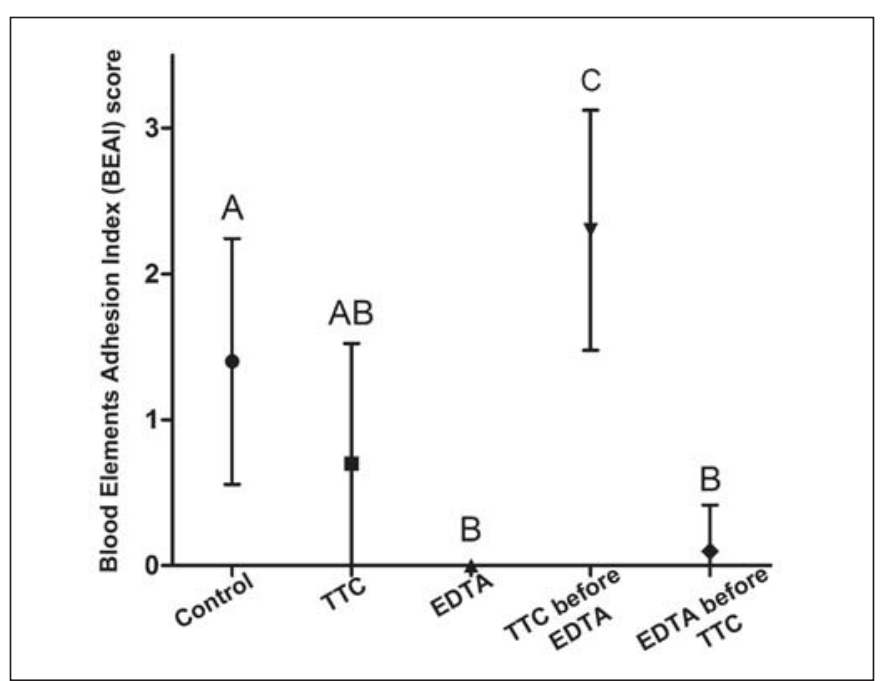

Fig. 3 - Blood clot stabilization on root dentin conditioned by tetracycline and/or EDTA. Results of blood elements adhesion index (BEAl) comparison between groups by ANOVA and Tukey's tests (mean and standard deviation). Groups with different letters had statistically significant differences $(p<0.05)$.

layer is composed by very small particles of organic and inorganic material varying in size from less than $1 \mu \mathrm{m}$ to over $15 \mu \mathrm{m}$. This layer is in intimate contact with the tooth surface and it is only removed by applying a demineralizing solution ${ }^{14}$. Smear layer removal by conditioning agents promotes collagen union between the exposed root surface fibrils and soft tissue collagen fibrils ${ }^{15}$. The binding of the two sources of collagen by fibronectin in the early stages of wound repair inhibits the downgrowth of oral epithelium and enhances fibroblast chemotaxis, migration and attachment ${ }^{15}$. In this way, clinical outcome tends to improve with more consistent results.

TTC became one of the most widely used and studied demineralizing agent since in vitro studies ${ }^{16}$ suggested its potential use in regenerative procedures. TTC is an antibiotic with affinity for mineralized tissues; it is adsorbed and then slowly released to surrounding tissues for up to 14 days. Many concentrations and application times were tested ranging from $0.5 \%$ to $200 \%$ and from 0.5 to $10 \mathrm{~min}^{17}$.

The low $\mathrm{pH}$ of the saturated solutions of TTC has been suggested as one of the reasons for the reduced cellular insertion and for the unpredictable results, as it could denature the organic matrix of dentin ${ }^{16}$. It was also suggested that acid etching would interfere on periodontal healing by its necrotizing effect on the surrounding progenitor cells ${ }^{5}$.

Table 1: Frequency distribution of scores according to blood elements adhesion index (BEAI)

\begin{tabular}{|c|c|c|c|c|c|}
\hline & $\begin{array}{c}\text { Control (GI) } \\
n(\%)\end{array}$ & $\begin{array}{c}\text { TTC (GII) } \\
n(\%)\end{array}$ & $\begin{array}{c}\text { EDTA (GIII) } \\
n(\%)\end{array}$ & $\begin{array}{c}\text { TTC+EDTA (GIV) } \\
n(\%)\end{array}$ & $\begin{array}{c}\text { EDTA+TTC (GV) } \\
n(\%)\end{array}$ \\
\hline Score 0 & $2(20)$ & $5(50)$ & $10(100)$ & $0(0)$ & $9(90)$ \\
\hline Score 1 & $2(20)$ & $3(30)$ & 0 & $2(20)$ & $1(10)$ \\
\hline Score 2 & $8(60)$ & $2(20)$ & 0 & $3(30)$ & 0 \\
\hline Score 3 & $0(0)$ & 0 & 0 & $5(50)$ & 0 \\
\hline Total & 10 & 10 & 10 & 10 & 10 \\
\hline
\end{tabular}

Score 0: Absence of fibrin network and blood cells; score 1: scarce fibrin and cells; score 2: moderate cells and thin fibrin network; score 3: dense fibrin with blood cells; TTC: tetracycline; EDTA: ethylenediaminetetraacetic acid 
EDTA was used in this study to investigate the effect of non-acidic substances to stabilize clot. Our findings demonstrated that 24\% EDTA failed to produce a compatible root surface for clot stabilization, corroborating previous studies ${ }^{18-19}$. All samples in this group had no attached cells or fibrin. EDTA at $12 \%-24 \%$ concentrations with neutral $\mathrm{pH}$ was introduced aiming to remove the smear layer without damaging biological structures ${ }^{20}$. Notwithstanding, some authors ${ }^{8}$ presented reliable findings using monoclonal antibodies and field emission in-lens scanning electron microscopy (FEISEM) showing that EDTA treatment is able to etch and expose collagen fibrils and proteoglycans without any degradation of dentin collagen matrix. It is speculated whether EDTA residues interfere on clot stabilization.

A meta-analysis study suggested that guided tissue regeneration-based root coverage with root conditioning could be used successfully to repair gingival recession defects ${ }^{21}$. According to those results, the use of absorbable membranes and root conditioning, resulted in significantly improved percentages of sites with complete root coverage. Clot stabilization seen in the present results may be important as a scaffold for the penetration and proliferation of the surrounding tissues.

In this study, EDTA improved TTC (GIV) results increasing the amount of samples with score 3 (dense fibrin network with presence of blood cells). A possible explanation is that TTC action by low pH denatures the organic matrix of dentin and using EDTA as a neutralizing agent, probably avoids an intense dentin demineralization, preserving a collagen scaffold for clot stabilization and proliferation of connective tissue cells ${ }^{22}$. Clot formation and adhesion are the initial steps of wound healing after periodontal therapy. A rapid adherence of the clot to root surface acts as a physical barrier reducing epithelial apical migration and avoiding a long junctional epithelium formation ${ }^{4}$.

Acid conditioning agents such as citric acid and TTC cause the acidification of extracellular medium, which induces cell death, and some authors suggest that $\mathrm{pH}$ elevation can prevent or reduce tissue damage 5 . The use of EDTA after TTC as a neutralizing agent showed promising results in clot organization. In vivo studies should be carried out to verify whether this combination maintains the connective tissue cells more viable to adhere on root surface and if the slow release of TTC after therapy is maintained. A previous study already tested the combination of TTC with citric acid as subgingival irrigants after root scaling and planing ${ }^{23}$. The authors found a synergistic effect of the two agents and a more powerful demineralizing potential than TTC-containing gel.

The only systematic review about this subject indicated no significant clinical effect of root conditioning on tissue repair $^{11}$. However, the author highlighted that several factors such as lack of controls, non-calibrated and non-blinded examiners, and small sample sizes reduced the quality of most studies. Thus, the author stated that the conclusions of his review must be critically considered ${ }^{11}$.

It was concluded that compared with TTC alone, the use of EDTA after TTC in an attempt to neutralize its acid
$\mathrm{pH}$, improved clot formation and organization in vitro, indicating that this may be a promising therapy. More clinical studies are required to verify the effects of root conditioning as an adjunct to conventional periodontal treatment.

\section{Acknowledgements}

This study was supported by The São Paulo State Foundation - FAPESP (grants 03/04252-2 and 03/04754-8).

\section{References}

1. Theodoro LH, Zezell DM, Garcia VG, Haypek P, Nagata MJ, de Almeida $\mathrm{JM}$, et al. Comparative analysis of root surface smear layer removal by different etching modalities or erbium:yttrium-aluminum-garnet laser irradiation. Ascanning electron microscopy study. Lasers Med Sci. 2010; 25: 485-91.

2. Adriaens PA, Adriaens LM. Effects of nonsurgical periodontal therapy on hard and soft tissues. Periodontol 2000. 2004; 36: 121-45.

3. Blomlof J, Lindskog S. Root surface texture and early cell and tissue colonization after different etching modalities. Eur J Oral Sci. 1995; 103: 17-24.

4. Leite FR, Moreira CS, Theodoro LH, Sampaio JE. Blood cell attachment to root surfaces treated with EDTA gel. Braz Oral Res. 2005; 19: 88-92.

5. Zandim DL, Leite FR, da Silva VC, Lopes BM, Spolidorio LC, Sampaio JE. Wound healing of dehiscence defects following different root conditioning modalities: an experimental study in dogs. Clin Oral Investig. 2013; 17 1585-93.

6. Leite FR, Nascimento GG, Leite ER, Leite AA, Sampaio JE. Effect of the association between citric acid and EDTA on root surface etching. J Contemp Dent Pract. 2013; 14: 5.

7. Fardal O, Lowenberg BF. A quantitative analysis of the migration, attachment, and orientation of human gingival fibroblasts to human dental root surfaces in vitro. J Periodontol. 1990; 61: 529-35.

8. Ruggeri A Jr, Prati C, Mazzoni A, Nucci C, Di Lenarda R, Mazzotti G, et al. Effects of citric acid and EDTA conditioning on exposed root dentin: An immunohistochemical analysis of collagen and proteoglycans. Arch Oral Biol. 2007; 52: 1-8.

9. Gamal AY, Mailhot JM. The effects of EDTAgel conditioning exposure time on periodontitis-affected human root surfaces: surface topography and PDL cell adhesion. J Int Acad Periodontol. 2003; 5: 11-22.

10. Dantas AA, Fontanari LA, Ishi Ede P, Leite FR, Zandim DL, Rached RS et al. Blood cells attachment after root conditioning and PRP application: an in vitro study. J Contemp Dent Pract. 2012; 13: 332-8.

11. Mariotti A. Efficacy of chemical root surface modifiers in the treatment of periodontal disease. Asystematic review. Ann Periodontol. 2003; 8: 205-26.

12. Leite FR, Sampaio JE, Zandim DL, Dantas AA, Leite ER, Leite AA. Influence of root-surface conditioning with acid and chelating agents on clot stabilization. Quintessence Int. 2010; 41: 341-9.

13. Baker DL, Stanley Pavlow SA, Wikesjo UM. Fibrin clot adhesion to dentin conditioned with protein constructs: an in vitro proof-of-principle study. J Clin Periodontol. 2005; 32: 561-6.

14. Cavassim R, Leite FR, Zandim DL, Dantas AA, Sampaio JE. Smear layer removal for collagen fiber exposure after citric acid conditionings. J Contemp Dent Pract. 2010; 11: E001-8.

15. Heritier M. Effects of phosphoric acid on root dentin surface. A scanning and transmission electron microscopic study. J Periodontal Res. 1984; 19 : 168-76.

16. Amaral NG, Rezende ML, Hirata F, Rodrigues MG, Sant'ana AC, Greghi $\mathrm{SL}$, et al. Comparison among four commonly used demineralizing agents for root conditioning: a scanning electron microscopy. J Appl Oral Sci. 2011; 19: 469-75. 
17. Isik AG, Tarim B, Hafez AA, Yalcin FS, Onan U, Cox CF. A comparative scanning electron microscopic study on the characteristics of demineralized dentin root surface using different tetracycline $\mathrm{HCl}$ concentrations and application times. J Periodontol. 2000; 71: 219-25.

18. Minocha T, RahulA. Comparison of fibrin clot adhesion to dentine conditioned with citric acid, tetracycline, and ethylene diamine tetra acetic acid: An in vitro scanning electron microscopic study. J Indian Soc Periodontol. 2012; 16: 333-41.

19. Preeja C, Janam P, Nayar BR. Fibrin clot adhesion to root surface treated with tetracycline hydrochloride and ethylenediaminetetraacetic acid:Ascanning electron microscopic study. Dent Res J (Isfahan). 2013; 10: 382-8.

20. Bogle G, Garrett $S$, Crigger M, Egelberg J. New connective tissue attachment in beagles with advanced natural periodontitis. J Periodontal Res. 1983; 18: 220-8.

21. Al-Hamdan K, Eber R, Sarment D, Kowalski C, Wang HL. Guided tissue regeneration-based root coverage: meta-analysis. J Periodontol. 2003; 74: 1520-33.

22. Fontanari LA, Pinto SC, Cavassim R, Spin-Neto R, Ishi Ede P, Sampaio $\mathrm{JE}$. Influence of dental exposure to oral environment on smear layer removal and collagen exhibition after using different conditioning agents. Braz Dent J. 2011; 22: 479-85.

23. George RP, Kumar S, Ramakrishna T, Emmadi P, Ambalavanan N. Effects of tetracycline-containing gel and a mixture of tetracycline and citric acid-containing gel on non-surgical periodontal therapy. Indian J Dent Res. 2013; 24: 52-9. 